\title{
Direct experimental observation of nonclassicality in ensembles of single-photon emitters
}

\author{
E. Moreva, ${ }^{1}$ P. Traina, ${ }^{1}$ J. Forneris, ${ }^{2}$ I. P. Degiovanni, ${ }^{1}$ S. Ditalia Tchernij,,${ }^{3,2}$ F. Picollo, ${ }^{3,2}$ G. Brida, ${ }^{1}$ \\ P. Olivero, ${ }^{3,2}$ and M. Genovese ${ }^{1,2}$ \\ ${ }^{1}$ Istituto Nazionale di Ricerca Metrologica, Strada delle cacce 91, 10135 Torino, Italy \\ ${ }^{2}$ Istituto Nazionale di Fisica Nucleare (INFN) Sez. Torino, 10125 Torino, Italy \\ ${ }^{3}$ Physics Department and "NIS" inter-departmental Centre-University of Torino, 10125 Torino, Italy \\ (Received 19 May 2017; revised manuscript received 7 November 2017; published 27 November 2017)
}

\begin{abstract}
In this work we experimentally demonstrate a recently proposed criterion addressed to detect nonclassical behavior in the fluorescence emission of ensembles of single-photon emitters. In particular, we apply the method to study clusters of nitrogen-vacancy centers in diamond characterized with single-photon-sensitive confocal microscopy. Theoretical considerations on the behavior of the parameter at any arbitrary order in the presence of Poissonian noise are presented and, finally, the opportunity of detecting manifold coincidences is discussed.
\end{abstract}

DOI: 10.1103/PhysRevB.96.195209

\section{INTRODUCTION}

One of the most debated issues in quantum mechanics is related to understanding the boundary separating the counterintuitive behavior of the systems governed by the quantum laws from the classical, familiar properties of the macroscopical systems. This transition also manifests itself in the realm of optics [1] where, even if undoubtedly the radiation emitted by any possible source of light is indeed composed by an ensemble of individual photons, the properties of classical sources differ consistently from those of nonclassical ones. In particular, single-photon sources (SPSs) have found many experimental and reliable realizations in systems such as heralded sources based on parametric down-conversion [2-9], quantum dots [10,11], trapped ions [12], molecules [13], and color centers in diamond [14-21]. Since nonclassical optical states have now become a fundamental resource for quantum technology [22,23], the determination of nonclassicality [24] for a state is not only important for studies concerning boundaries from quantum to classical world, but also represents an important tool for quantifying such resources. Vast literature exists on the characterization of SPSs [25]. Most of the techniques rely on the sampling of the second-order autocorrelation function (or Glauber function)

$$
g^{(2)}(\tau=0)=\left.\frac{\langle I(t) I(t+\tau)\rangle}{\langle I(t)\rangle\langle I(t+\tau)\rangle}\right|_{\tau=0},
$$

whose value is never smaller than 1 for classical light, while it is lower than 1 for sub-Poissonian light, and in particular vanishes for single-photon states, where $g^{(2)}(0)=0$ is expected in the ideal case. This quantity has been shown to be substantially equivalent to the parameter $\alpha$ introduced by Grangier et al. [26] (and throughout the paper we will refer to this parameter as $g^{(2)}$ without distinction), which is experimentally measured as the ratio between the coincidence probability at the ouput of a Hanbury Brown and Twiss interferometer (HBT) [27], typically a 50:50 beam splitter connected to two non-photon-number-resolving (non-PNR) detectors, and the product of the click probabilities at the two detectors [22]. This parameter can be generalized to account for the statistical properties of $N$-fold coincidence events at the outputs of detector-tree apparatuses and several techniques for the reconstruction of optical states as well as quantum enhanced imaging techniques are allowed by the experimental sampling of $g^{(N)}$ functions [28-44]. Unfortunately, the amount of background light can affect the measurement, leading to a camouflage of the quantum characteristics due to noise. More specifically, in practical cases, when sampling $g^{(2)}(0)$ to characterize single emitters it is not possible to distinguish between the true quantum signal and background light contribution and, in extreme cases, one is not able to detect a single emitter drowned in dominant noise bath. Recently a novel criterion allowing one to reveal no-classical light from large numbers of independent SPSs has been proposed [45]. According to the theoretical predictions, an experimental implementation of this criterion would be extremely advantageous not only because it would allow one to spot nonclassical signatures in the emission of clusters of emitters, but also because it can be shown that this technique is extremely robust in the presence of Poissonian noise, the parameter under test being absolutely independent from this kind of noise contribution (even when it is dominant).

In this work we experimentally apply the criterion [45] to directly detect nonclassical emission from ensembles of SPSs based on nitrogen-vacancy (NV) centers in nanodiamond observed by means of a confocal microscope coupled to four non-PNR single-photon detectors in a detector-tree configuration. Although the reported methodology can be generally extended to a broad range of different physical systems, NV defects in diamond have been elected to benchmark this new criterion. This choice is motivated by the high relevance of this physical system in quantum optics due to its appealing spin-dependent transition structure, as widely demonstrated by a broad range of works on the subject in recent years [46]. Moreover, the choice is motivated by the fact that generally the quantum-optical characterization of individual photon emitters in solid-state systems (such as NV and other color centers in diamond) is significantly affected by complex issues in the correct assessment of sources of background luminescence in confocal microscopy, such as nearby defects, scattered light, diffused fluorescence from extended defects, ambient light, etc. [14,17], which are not always easily manageable, or duly taken into account.

\section{THEORETICAL MODEL}

In general, the system considered here is an ensemble of $M$ single-photon emitters, each coupled to the detection 
system with an efficiency $\eta_{\alpha}(\alpha=1, \ldots, M)$, detected by $N$ non-PNR detectors connected by a generalized $N$-dimensional beam splitter (BS). Each detection channel has an overall efficiency (due to BS unbalance and detector efficiency) $\xi_{i}$ $(i=1, \ldots, N)$. Generally, $n$ incoming photons entering in the detector tree are distributed in the $N$ channels following the multinomial probability $\frac{n !}{\prod_{i=1}^{N} k_{i} !}\left(\frac{1}{N}\right)^{n}$ corresponding to $k_{i}$ photons in the $i$ th channel (satisfying $\sum_{i} k_{i}=n$ ). In each channel of the detector tree, the probability of observing a no-click event given $k_{i}$ photons is $\left(1-\xi_{i}\right)^{k_{i}}$, thus the click probability is $1-\left(1-\xi_{i}\right)^{k_{i}}$, since the positive operator-valued measurements (POVM) of a non-PNR single-photon detector at the end of each channel of the detector tree are

$$
\begin{gathered}
\hat{Q}_{\text {click }}=\sum_{n=0}^{+\infty}\left[1-\left(1-\xi_{i}\right)^{n}\right]|n\rangle\langle n|, \\
\hat{Q}_{\text {no-click }}=\sum_{n=0}^{+\infty}\left(1-\xi_{i}\right)^{n}|n\rangle\langle n| .
\end{gathered}
$$

Starting from this, it is possible to define the POVM of the single detection of the detector tree as

$$
\begin{gathered}
\hat{Q}_{[i]}^{[\text {Single }]}(0)=\sum_{n=0}^{+\infty} Q_{[i]}^{[\text {Single }]}(0 \mid n)|n\rangle\langle n|, \\
\hat{Q}_{[i]}^{[\text {Single }]}(1)=\hat{I}-\hat{Q}_{[i]}^{[\text {Single }]}(0),
\end{gathered}
$$

where $Q_{[i]}^{[\text {Single] }}(0 \mid n)=\left(1-\xi_{i} / N\right)^{n}$ is the probability that 0 out of $n$ incoming photons are detected per excitation pulse. Since the measurement is phase insensitive, the operators have diagonal form in the Fock basis and, due to the nature of non-PNR detectors (able only to distinguish between dark and light) the possible outcomes are " 0 " (the detector does not click) and " 1 " (the detector clicks).

Analogously, one can obtain the POVM associated to the no-click in all the outputs of the detector tree as

$$
\hat{Q}^{[\otimes N]}(0)=\sum_{n=0}^{+\infty} Q^{[\otimes N]}(0 \mid n)|n\rangle\langle n|,
$$

where $Q^{[\otimes N]}(0 \mid n)=\left(1-\frac{\sum_{i=1}^{N} \xi_{i}}{N}\right)^{n}$.

Finally, the POVM of $N$-fold coincidence results in

$$
\hat{Q}^{[\otimes N]}(N)=\sum_{n=0}^{+\infty} Q^{[\otimes N]}(N \mid n)|n\rangle\langle n|,
$$

where $Q^{[\otimes N]}(N \mid n)$ has in general a nontrivial form, but under the hypothesis that the detection system is a tree of perfectly balanced identical detectors $\left(\xi_{i}=\xi, \forall i\right)$, it reduces to

$$
Q^{[\otimes N]}(N \mid n)=\sum_{r=0}^{N}(-1)^{r} \frac{N !}{r !(N-r) !}\left(1-\frac{r \xi}{N}\right)^{n} .
$$

The latter assumption of perfectly balanced detector- tree does not limit the general validity of the discussion because it can be shown that also without this hypothesis the technique preserves its noise-resilience properties (see Appendix D).

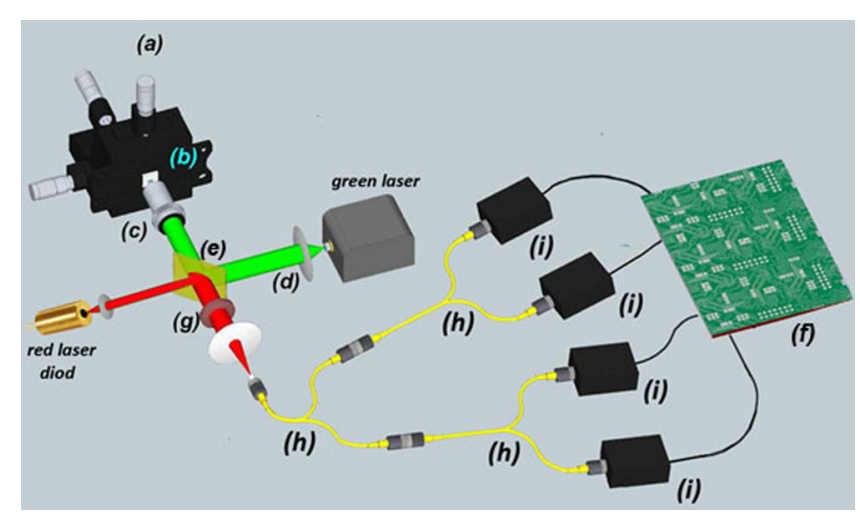

FIG. 1. Experimental setup: (a) XYZ piezoelectric stage, (b) sample, (c) oil immersion objective, (d) excitation light, (e) dichroic mirror, (f) coincidence electronics, (g) long-pass filters, (h) 50:50 beam splitter, and (i) single-photon detectors.

The generalized $g^{(N)}(0)$ function is thus expressed in terms of detection probabilities as

$$
g^{(N)}(0)=\frac{P_{\text {click }^{\otimes N}}}{\prod_{i=1}^{N} P_{\text {click }[i]}},
$$

where $P_{\text {click }}^{\otimes N}=\operatorname{tr}\left[\hat{\rho} \hat{Q}^{[\otimes N]}(N)\right]$ is the probability of $N$-fold coincidence at the output of the detector tree, and $P_{\operatorname{click}[i]}=$ $\operatorname{tr}\left[\hat{\rho} \hat{Q}_{[i]}^{[\text {Single }]}(1)\right]$ is the probability for the $i$ th detector to fire, $\hat{\rho}$ being the density matrix describing the quantum state of the ensemble of emitters. As stated above, the condition $g^{(N)}(0)=1$ can be adopted to discriminate between classical and nonclassical states, while ideally $g^{(N)}(0)=0$ for any order of $N$ for single-photon states. Instead, the nonclassicality criterion under study [45] is expressed by the fact that for any classical system the following proposition is verified:

$$
\theta^{(N)}(0)=\frac{P_{0^{\otimes N}}}{\prod_{i=1}^{N} P_{0[i]}}>1,
$$

where $P_{0[i]}=\operatorname{tr}\left[\hat{\rho} \hat{Q}_{[i]}^{[\text {Single }]}(0)\right]$ is the no-click probability at the $i$ th detector and $P_{0^{\otimes N}}=\operatorname{tr}\left[\hat{\rho} \hat{Q}^{[\otimes N]}(0)\right]$ is the probability that all the $N$ detectors of the detector-tree do not click in correspondence of an excitation event.

\section{EXPERIMENTAL SETUP}

We perform the comparison of the behaviors of $g^{(2)}$ and $\theta^{(2)}$ exploiting different ensembles of single-photon emitters with different levels of background Poissonian noise in synthetic Ib nanodiamond (ND) powders produced by ElementSix by fragmentation of high-pressure-high-temperature (HPHT) synthetic diamond (see Appendix A). The sample is observed via a single-photon-sensitive confocal microscope connected to a detector-tree configuration of four detectors (Fig. 1). The excitation light is provided by a solid-state laser at $532 \mathrm{~nm}$ (PICOQUANT LDH-PFA-530L) in pulsed regime $(5 \mathrm{MHz}$ repetition rate, $50 \mathrm{ps}$ FWHM) whose output is coupled into a single-mode fiber and then collimated by a $4 \times$ objective. A dichroic mirror (long-pass at $570 \mathrm{~nm}$ ) reflects the excitation light (3 $\mathrm{mW}$ maximum) inside the oil immersion objective (Olympus, $100 \times, \mathrm{NA}=1.3$ ) focusing inside the sample and transmits the fluorescence light $(640-800 \mathrm{~nm}$, coupled by the 


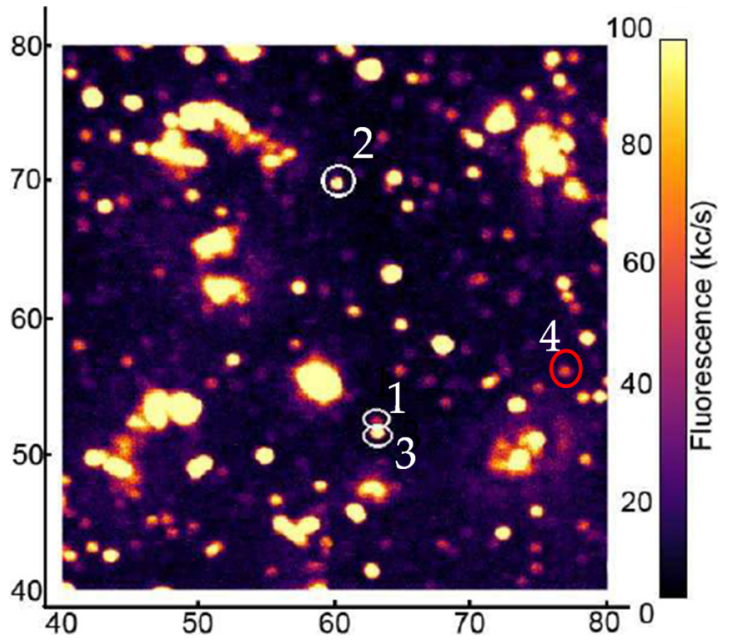

FIG. 2. Typical fluorescence map of the selected area of the sample obtained with the single-photon-sensitive confocal microscope. The three highlighted spots correspond to the objects under study. The acquisition software used is "Qudi," developed at Ulm University [48].

same objective) toward the detecting apparatus. A closed-loop $\mathrm{XYZ}$ piezoelectric stage, remotely controlled via PC, allows submicrometric-resolution positioning in an $80 \mu \mathrm{m} \times 80 \mu \mathrm{m}$ area. A long-pass filter allows obtaining a suitable attenuation of the pump component. Then, the signal is focused by an $f=100 \mathrm{~mm}$ achromatic doublet and coupled to a $50 \mu \mathrm{m}$ multimode optical fiber. The fiber leads to a detector-tree configuration realized by means of three integrated 50:50 beam splitters in cascade connecting to four single photon avalanche photodiodes (Perkin-Elmer SPCM-AQR), operating in Geiger mode. This configuration, reproducing six nonmutually independent HBT interferometers (or equivalently two completely independent HBT interferometers) [27], allows the detection of all the twofold coincidences among the detection channels and to obtain six estimates of the second-order autocorrelation functions $\left(g^{(2)}\right)$. The signal counts and coincidences are measured via an Id Quantique ID800 time-to-digital converter. The pulses (60 ps FWHM) of the laser simulating the Poissonian noise (PICOQUANT LDH-D-C-690), emitting at $685 \mathrm{~nm}$, inside the detection window, were electronically synchronized with the excitation laser emission. This laser was directly coupled to the pinhole of the detection system.

\section{RESULTS}

We implemented the measurement of $g^{(2)}$ and $\theta^{(2)}$ by the characterization of three fluorescent objects in a nanodiamond sample (see Fig. 3). To perform this study, the width of the time window ( $40 \mathrm{~ns}$ ) was chosen to be compatible with the lifetime of the centers (around $25 \mathrm{~ns}$ ). The objects under study (see Fig. 2) are dubbed Item-1, Item-2, and Item-3. Item- 1 is not far from a single-photon emitter having $g^{(2)}$ value below 0.5 $\left[g_{I_{1}}^{(2)}(0)=0.407 \pm 0.012\right]$ [47] if no artificial noise is added, while Item- 2 and Item-3 are clusters of unknown quantities of single-photon emitters [respectively, $g_{I_{2}}^{(2)}(0)=0.832 \pm 0.004$ and $g_{I_{3}}^{(2)}(0)=0.66 \pm 0.01$, always without noise]. The overall efficiency of the system has been estimated as (1.582 \pm
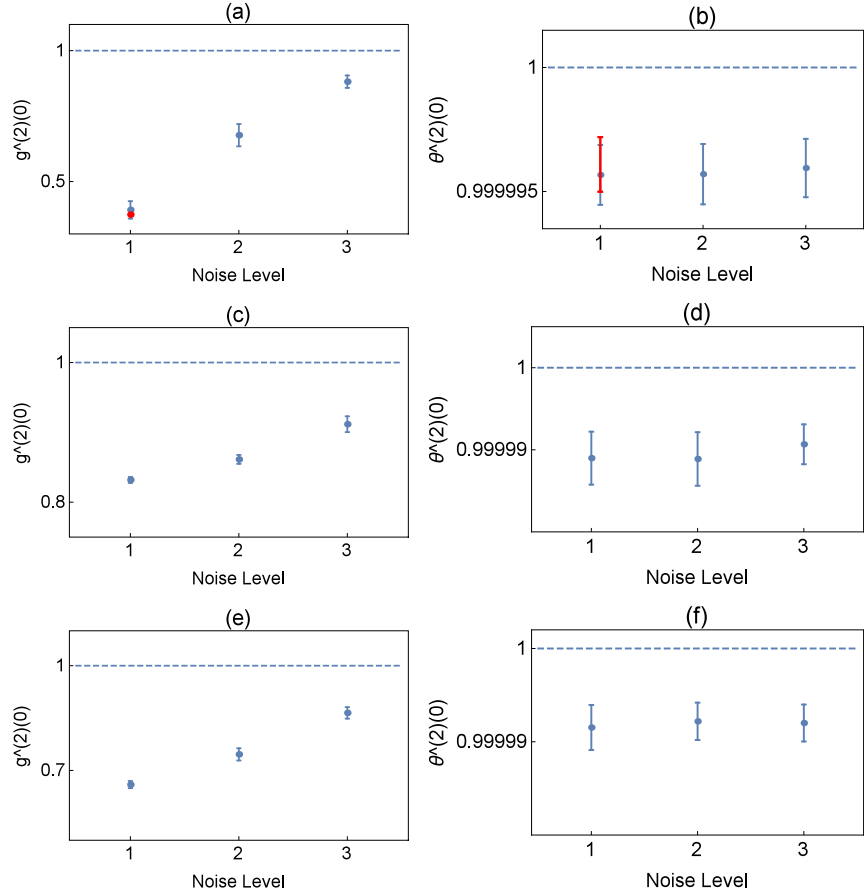

FIG. 3. (a) Plot of $g_{I_{1}}^{(2)}(0)$. (b) Plot of $\theta_{I_{1}}^{(2)}(0)$. (c) Plot of $g_{I_{2}}^{(2)}(0)$. (d) Plot of $\theta_{I_{2}}^{(2)}(0)$. (e) Plot of $g_{I_{3}}^{(2)}(0)$. (f) Plot of $\theta_{I_{3}}^{(2)}(0)$. All values were measured for three different levels of Poissonian noise (1: noise off; 2: 10000 counts/s due to noise; 3: 25000 counts/s due to noise). Each measure is the average of ten runs, coincidences registered in every twofold combination of the detector-tree branches, of $200 \mathrm{~s}$. Excitation rate is $5 \mathrm{MHz}$. The red dot in (a) and (b) corresponds to a successive repetition of the first measurement to test the stability of the apparatus.

$0.002) \%$ (calculated as the ratio between the emission rate of Item- 1 and the excitation rate). In order to simulate Poissonian noise, a laser source at a wavelength falling in the detection spectral window was reflected directly in the coupling pinhole of the microscope. To analyze the robustness of the two parameters against noise, measurements on each sample were performed in the absence of noise and in the presence of two different values of intensity of the noise source. These two noise levels, estimated in terms of counts/s observed by the HBT system as a whole, in the absence of single-photon source emissions are 10000 counts/s and 25000 counts/s.

For testing the stability of the experimental system, the measurement without Poissonian noise was repeated after the measurement including noise and the $g$ and $\theta$ parameters are found to be consistent with the first measurements (Fig. 3). As an example, this measurement in the case of Item- 1 is shown as a red dot. Also, to test the capability of our setup to detect nonclassical behavior, we performed the measurement of $\theta^{(2)}$ and $g^{(2)}$ parameters on the light reflected by a nonfluorescent nanodiamond present in the sample (Item-4 in Fig. 2). This kind of object is not distinguishable from the emitters in a confocal map but does not produce antibunching (being produced by coherent light) and can be recongnized only from its spectral features. As expected, this object showed clear signatures of classical emission $\left[1-g^{(2)}(0)=0.004 \pm 0.005\right.$, $\left.1-\theta^{(2)}(0)=(-4 \pm 2) * 10^{-8}\right]$. 


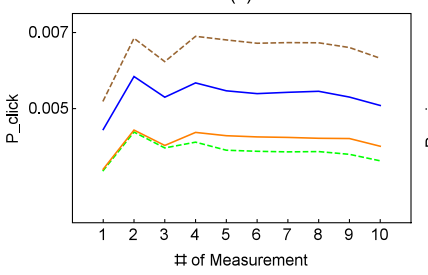

(c)

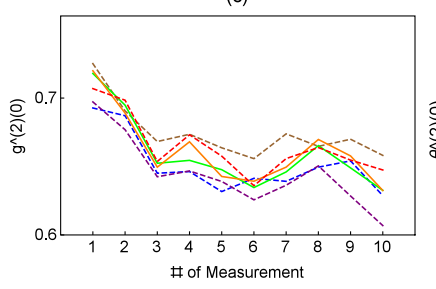

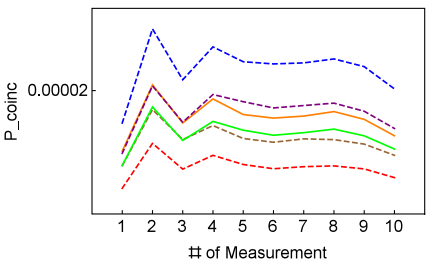

(d)

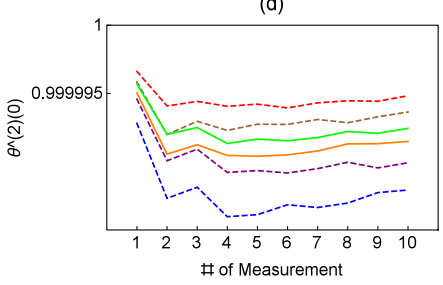

FIG. 4. (a) Click probabilities at the four outputs of the detectortree while observing Item-3. The style of the line (continuous/dashed) distinguishes between the outputs of the two independent BS constituting the detector-tree. (c) and (d) values, respectively, of $g^{(2)}(0)$ and $\theta^{(2)}(0)$ measured for each twofold combination among the four outputs of the detector-tree while observing Item-3. Same measurement settings as Fig. 3. In (b)-(d) the continuous lines mark the two independent twofold coincidences.

\section{DISCUSSION}

As expected from the analysis in Ref. [45], the experimental data (Fig. 3) clearly demonstrate the advantages of the nonclassicality criterion based on $\theta^{(2)}$ with respect to $g^{(2)}$ : firstly, the parameter estimation is more robust against Poissonian noise since the three $\theta^{(2)}$ values for each object are perfectly compatible, while $g^{(2)}$ values differ considerably for different noise contributions; secondly, the deviation from classicality is even stronger when the object under study is not a single emitter but instead an ensemble of them. This is supported by two observations: (i) the deviation from the classical value in terms of $\theta^{(2)}$ is larger for clusters characterized by a greater $g^{(2)}$ value with respect to the others (measured in the same conditions); (ii) the raw data regarding the two-, three- and fourfold coincidence events (not reported here) show significantly different multiphoton coincidence behavior for the three objects. Those events are rare for Item-1, they are significantly occurring for Item-3, and they are definitely more frequent for Item-2, justifying the conclusion that the number of centers in each cluster increases accordingly. For this reason this method allows detecting nonclassical behavior of quantum emitters without the necessity of isolating a single one. On the other hand, the $\theta^{(2)}$ function presents some disadvantages, since its value depends strongly on the efficiency of the channel $\xi$ (including detection efficiency and splitting ratio of the detector-tree; see the Appendices) as opposed to $g^{(2)}$ which is independent from the balance of the branches of the detector tree. This can be observed in the plots in Fig. 4, where respectively, the click probabilities of the single channels [inset (a)], the twofold coincidence probabilities [inset (b)], and the experimental $g^{(2)}$ [inset (c)] and $\theta^{(2)}$ [inset (d)] values in the characterization of Item-3 are shown. It results that the unbalance of the detector tree in terms of detection efficiency which is observable in Fig. 4(a), is compensated when sampling $g^{(2)}$ [Fig. 4(c)],

delivering six fairly consistent estimates, while the values of $\theta^{(2)}$ obtained for different pairs of channels are not consistent [Fig. 4(d)]. This results in a rather large uncertainty (calculated as the standard deviation of the six sampled quantities) in the mean $\theta^{(2)}$ values plotted in Figs. 3(b), 3(d) and 3(e). One must be reminded anyway that the average of the latter values is below the classical limit within a $3 \sigma$ confidence level [as can be observed in Fig. 3(f)] thus this inconsistency does not affect the observation of nonclassical behavior. See Tables III and IV of Appendix B for data collected by a single beam splitter (showing smaller uncertainty).

In our experiment we limited the analysis to the second order of correlation of $\theta$ and $g$ functions. Since the family of nonclassicality criteria under test is defined for any arbitrary order, it is reasonable to investigate the possible advantages/disadvantages of experimentally sampling threeand fourfold coincidences as well. The calculations on the behavior of the $\theta^{(N)}$ functions clearly reveal the independence of the value of the parameter with respect to the noise level for all the orders of correlation, exactly as we observed in the second-order case and at variance with the behavior of $g$ functions (see Appendix C). Even if our detection apparatus is capable of detecting up to fourfold coincidences, for the three objects under study the three- and fourfold coincidence rates were extremely low (respectively, $10^{-7}$ and $10^{-9}$; less than the associated statistical uncertainty for the brightest object, Item-2), so that no significant consideration on high-order behavior could be extracted in our experimental conditions.

\section{SUMMARY AND CONCLUSIONS}

In conclusion, in this paper we experimentally demonstrated the advantage of nonclassicality criteria based on $\theta^{(N)}$ function proposed in Ref. [45] compared to $g^{(N)}$ characterization. The most important of these advantages is the resilience to Poissonian noise affecting the source. This technique has the drawback that the $\theta^{(N)}$ value depends on the experimental conditions (optical and coupling losses, detection efficiency, detector-tree splitting ratio). On the other hand, if the measurement apparatus is previously characterized, one could identify the number of single-photon emitters in the ensemble and then optimization can be carried on to remove the noise source. Another important advantage of $\theta^{(N)}$ measurement is that its value gets more nonclassical for increasing number of emitters, while $g^{(N)}$ approaches classicality in this cases. The technique is of high interest in quantum technology because, in principle, the information gained by its implementation can be used together with other multicoincidence techniques [32-44], leading to an improvement of the latter applications. Finally, for more complex cases (not only single emitters and Poissonian noise but also thermal modes are present), $\theta^{(N)}$ and $g^{(N)}$ measurements can be possibly combined as in the algorithm described in Ref. [28] to obtain a rigorous and reliable reconstruction of the modal structure of the fields.

Our results pave the way both for studying quantumclassical boundary and for quantifying resources needed in quantum technologies.

Note added. Recently we became aware that another work produced similar results albeit considered from a different perspective [49]. 
TABLE I. Table of the experimental deviations of $g^{(2)}(0)$ from the classical limit.

\begin{tabular}{lccc}
\hline \hline $1-g^{(2)}(0)$ & Noise level 1 & Noise level 2 & Noise level 3 \\
\hline Item-1 & $0.61 \pm 0.03$ & $0.32 \pm 0.04$ & $0.12 \pm 0.02$ \\
Item-2 & $0.168 \pm 0.004$ & $0.139 \pm 0.006$ & $0.088 \pm 0.011$ \\
Item-3 & $0.34 \pm 0.01$ & $0.254 \pm 0.017$ & $0.135 \pm 0.016$ \\
Item-1 (check) & $0.63 \pm 0.03$ & & \\
\hline \hline
\end{tabular}

\section{ACKNOWLEDGMENTS}

This research activity was supported by the following projects: EMPIR-14IND05 271 "MIQC2" (the EMPIR initiative is cofunded by the EU H2020 272 and the EMPIR Participating States), MIUR Progetto 273 Premiale 2014 "Q-SecGroundSpace", "DIESIS" project funded by the Italian National Institute of Nuclear Physics (INFN)-CSN5 within the Young Research grant scheme, and coordinated research project F11020 supported by the International Atomic Energy Agency (IAEA). ND preparation was partly performed within the "Dia.Fab." experiment at the INFN-LNL laboratories, Italy. The PL mapping of ND samples was performed with the acquisition software Qudi developed at the Institute for Quantum Optics-University of Ulm. The support of the research group of Prof. F. Jelezko is gratefully acknowledged.

\section{APPENDIX A: SAMPLE PREPARATION}

This work was performed on synthetic nanodiamond (ND) powders produced by ElementSix by fragmentation of HPHT synthetic diamond with a nominal size of distribution comprised between 10 and $250 \mathrm{~nm}$. The powders were classified as Ib type, with a nominal substitutional $\mathrm{N}$ concentration of 10-100 ppm, and contained a low amount of native NV centers as a consequence of the high crystalline quality of the batch. NV centers were therefore fabricated through the introduction of radiation-induced vacancies and a subsequent thermal annealing [50]. The ND powders were first exposed to an acid bath $\left(\mathrm{H}_{2} \mathrm{SO}_{4}: \mathrm{HNO}_{3}=9: 1\right.$ solution; $72 \mathrm{~h}$ at $\left.100^{\circ} \mathrm{C}\right)$ to remove organic contaminations and graphite, and subsequently dispersed over a suitable substrate for ion irradiation. Then, they were irradiated with a $2 \mathrm{MeV} \mathrm{H}^{+}$beam at the AN2000 Accelerator of the INFN National Laboratories of Legnaro (Italy). An irradiation fluence of $5 \times 10^{12}$ protons $/ \mathrm{cm}^{2}$ was chosen, based on the ND median size and the NV centers creation efficiency [51], as the optimal condition to fabricate 150-nm-sized NDs statistically containing one individual NV center. Then, the powders were exposed to a thermal treatment $\left(800^{\circ} \mathrm{C}\right.$ for $1 \mathrm{~h}$, in an 800 -mbar controlled $N_{2}$ atmosphere) in order to promote the formation of $\mathrm{NV}$ centers. After the annealing process, an additional cleaning step was
TABLE III. Table of the experimental deviations of $g^{(2)}(0)$ from the classical limit as sampled by a single beam splitter of the detector tree.

\begin{tabular}{lccr}
\hline \hline $1-g^{(2)}(0)$ & Noise level 1 & Noise level 2 & \multicolumn{1}{c}{ Noise level 3 } \\
\hline Item-1 & $0.615 \pm 0.009$ & $0.345 \pm 0.011$ & $0.130 \pm 0.008$ \\
Item-2 & $0.166 \pm 0.003$ & $0.141 \pm 0.005$ & $0.0966 \pm 0.004$ \\
Item-3 & $0.34 \pm 0.03$ & $0.260 \pm 0.007$ & $0.144 \pm 0.004$ \\
\hline \hline
\end{tabular}

performed by a 30-min sonic bath in $\mathrm{H}_{2} \mathrm{SO}_{4}$, followed by a cleaning in Piranha solution $\left(\mathrm{H}_{2} \mathrm{SO}_{4}: \mathrm{H}_{2} \mathrm{O}_{2}=3: 1\right)$ to remove organical residuals and to dissolve metal oxides and carbonates contents from the ND powders. The samples were finally dispersed on coverslip glass substrates for the subsequent optical investigation.

\section{APPENDIX B: EXPERIMENTAL VALUES}

Tables I and II report the experimental deviations from the classicality $\left[1-g^{(2)}(0), 1-\theta^{(2)}(0)\right]$ experimentally measured as plotted in Fig. 3 .

Tables III and IV, instead, report the experimental deviations from the classicality $\left[1-g^{(2)}(0), 1-\theta^{(2)}(0)\right]$ as measured by a single beam splitter of the detector tree.

Finally, Table V shows the experimental count rates relative to the characterized objects for each detection channel.

\section{APPENDIX C: CALCULATION OF $\theta^{(N)}$ AND $g^{(N)}$ IN PRESENCE OF POISSONIAN NOISE}

According to the notation introduced in Sec. II, it follows that the probability $P_{0^{\otimes N}}=\operatorname{tr}\left[\hat{\rho} \hat{Q}^{[\otimes N]}(0)\right]\left(P_{0[i]}=\right.$ $\left.\operatorname{tr}\left[\hat{\rho} \hat{Q}_{[i]}^{[\text {Single] }}(0)\right]=P_{0}\right)$ in Eq. $(10), \hat{\rho}$ being the density matrix describing the quantum state of the ensemble of emitters, can be expressed in the form

$$
\sum_{n=0}^{\infty} \sigma^{n} p_{n}
$$

where $p_{n}=\langle n|\hat{\rho}| n\rangle$ is the probability distribution of the photons and $\sigma^{n}$ is equal to $(1-\xi)^{n}\left[\left(1-\frac{\xi}{N}\right)^{n}\right]$. We study the case of single emitters' fluorescence in the presence of Poissonian noise. The photon-number probability distribution in this case is

$$
p_{n}=\sum_{m=0}^{M} \sum_{k=0}^{\infty} \delta_{n, m+k} P_{s p s}(m) P_{\lambda}(k),
$$

where, assuming that all the emitters in the ensemble are coupled with the same efficiency $\left(\eta_{\alpha}=\eta, \forall \alpha\right), P_{s p s}(m)=$ $\frac{M !}{m !(M-m) !} \eta^{n}(1-\eta)^{M-m}$ is the distribution of the photons of

TABLE II. Table of the experimental deviations of $\theta^{(2)}(0)$ from the classical limit.

\begin{tabular}{lccc}
\hline \hline $1-\theta^{(2)}(0)$ & Noise level 1 & Noise level 2 & Noise level 3 \\
\hline Item-1 & $(4.3 \pm 1.2) \times 10^{-6}$ & $(4.3 \pm 1.2) \times 10^{-6}$ & $(4.1 \pm 1.2) \times 10^{-6}$ \\
Item-2 & $(11 \pm 3) \times 10^{-6}$ & $(11 \pm 3) \times 10^{-6}$ & $(9 \pm 2) \times 10^{-6}$ \\
Item-3 & $(8 \pm 2) \times 10^{-6}$ & $(8 \pm 2) \times 10^{-6}$ & $(8 \pm 2) \times 10^{-6}$ \\
Item-1 (check) & $(4 \pm 1) \times 10^{-6}$ & & \\
\hline \hline
\end{tabular}


TABLE IV. Table of the experimental deviations of $\theta^{(2)}(0)$ from the classical limit as sampled by a single beam splitter of the detector tree.

\begin{tabular}{lcrc}
\hline \hline $1-\theta^{(2)}(0)$ & Noise level 1 & Noise level 2 & Noise level 3 \\
\hline Item-1 & $(40 \pm 3) \times 10^{-7}$ & $(41 \pm 2) \times 10^{-7}$ & $(39 \pm 3) \times 10^{-7}$ \\
Item-2 & $(100 \pm 7) \times 10^{-7}$ & $(103 \pm 9) \times 10^{-7}$ & $(92 \pm 7) \times 10^{-7}$ \\
Item-3 & $(77 \pm 1.2) \times 10^{-7}$ & $(73 \pm 3) \times 10^{-7}$ & $(75 \pm 5) \times 10^{-7}$ \\
\hline \hline
\end{tabular}

the emitters, $P_{\lambda}(k)=\frac{\lambda^{k} e^{-\lambda}}{k !}$ is the distibution of the Poissonian light, and $\delta_{x, y}$ is the Kronecker delta. By substituting the suitable value for $\sigma$ in Eq. (C1), one gets

$$
\begin{gathered}
P_{0^{\otimes N}}=(1-\eta \xi)^{M} e^{-\lambda \xi}, \\
P_{0[i]}=P_{0}=\left(1-\frac{\eta \xi}{N}\right)^{M} e^{-\lambda \xi / N} .
\end{gathered}
$$

Finally, substituting Eqs. (C3) and (C4) in Eq. (10), the $\lambda$-dependent terms appear as equal factors both in the numerator and in the denominator of the ratio and are simplified, resulting in

$$
\theta^{(N)}(0)=\frac{(1-\eta \xi)^{M}}{\left(1-\frac{\eta \xi}{N}\right)^{M N}}
$$

Thus, under our assumptions, the parameter $\theta^{(N)}$ estimation is independent from the Poissonian contribution at any order $N$ (at variance with $g^{(N)}$ ).

This parameter must be compared with the $g^{(N)}$ function that is expressed according to Eq. (9). In order to calculate it in analogy with the expression of $\theta^{(N)}$, we must first of all write the probability of $N$-fold coincidence:

$$
\begin{aligned}
P_{\text {click }} \otimes N & =\operatorname{tr}\left[\hat{\rho} \hat{Q}^{[\otimes N]}(N)\right]=\sum_{n=0}^{\infty} Q^{[\otimes N]}(N \mid n) p_{n} \\
& =\sum_{r=0}^{N}(-1)^{r} \frac{N !}{r !(N-r) !}\left(1-\frac{\eta r \xi}{N}\right)^{M} e^{-\lambda r \xi / N},
\end{aligned}
$$

leading to

$$
\begin{aligned}
g^{(N)}(0) & =\frac{P_{\text {click }^{\otimes N}}}{\left(P_{\text {click }}\right)^{N}} \\
& =\frac{\sum_{r=0}^{N}(-1)^{r} \frac{N !}{r !(N-r) !}\left(1-\frac{\eta r \xi}{N}\right)^{M} e^{-\lambda r \xi / N}}{\left[1-\left(1-\frac{\eta \xi}{N}\right)^{M} e^{-\lambda \xi / N}\right]^{N}},
\end{aligned}
$$

where, in accordance with Eq. (C4) we used $P($ click $)=$ $1-P_{0}$. It is clear that, in opposition with the $\theta^{(N)}$ case,
TABLE V. Table of the experimental count rates at the four outputs of the detector tree (no noise added).

\begin{tabular}{lcccc}
\hline \hline Rate $(\mathrm{kHz})$ & Ch 1 & Ch 2 & Ch 3 & Ch 4 \\
\hline Item-1 & $22.4 \pm 0.2$ & $23.8 \pm 0.7$ & $16.90 \pm 0.04$ & $16.0 \pm 0.3$ \\
Item-2 & $57.1 \pm 1.2$ & $70 \pm 2$ & $40.5 \pm 0.8$ & $44 \pm 1$ \\
Item-3 & $37.3 \pm 1.9$ & $43 \pm 3$ & $27.8 \pm 1.4$ & $27.8 \pm 1.7$ \\
\hline \hline
\end{tabular}

the contribution of the Poissonian terms to $g^{(N)}$ cannot be eliminated.

\section{APPENDIX D: CALCULATION OF $\theta^{(N)}$ FOR UNBALANCED MULTIPORT HBT INTERFEROMETER IN PRESENCE OF POISSONIAN NOISE}

In the case of a nonideal (unbalanced) multiport HBT interferometer, the disproportion of the single-photon detections at each output port of the interferometer can be due either to the nonideal splitting ratio of the beam splitters of the detector tree at the heart of the multiport HBT or to the different value of quantum efficiency of each detector. To simplify the calculation we consider the situation in which the beam splitters are ideal (thus, for an $N$-port HBT interferometer, the probability of finding a single photon in each port is $1 / N$ ) and the whole unbalancement is summarized in the quantum efficiency $\xi_{i}$ of each detector. Starting from Eqs. (2)-(7), and carrying on the calculation, in complete analogy with Appendix $\mathrm{C}$ but without the assumption of identical detectors, we will obtain that

$$
\begin{gathered}
P_{0^{\otimes N}}=\left(1-\eta \frac{\sum_{i=1}^{N} \xi_{i}}{N}\right)^{M} e^{-\lambda\left(\sum_{i=1}^{N} \xi_{i} / N\right)}, \\
P_{0[i]}=P_{0}=\left(1-\frac{\eta \xi_{i}}{N}\right)^{M} e^{-\lambda \xi_{i} / N} .
\end{gathered}
$$

Thus, also in the case of an unbalanced HBT interferometer, $\theta^{(N)}(0)$ is independent from the Poissonian noise. There is essentially a trade-off between $\theta^{(N)}$ and $g^{(N)}$ measurements. While $g^{(N)}$ values are independent from inefficiencies and losses, and obviously from HBT interfermeter unbalancement, they are strongly affected by Poissonian noise. On the contrary, $\theta^{(N)}$ values strongly depend on inefficiencies and losses, but they are completely unaffected by the Poissonian noise irrespective of the level of unbalancement of the HBT interferometer.

\section{APPENDIX E: EXPLICIT $\theta^{(N)}$ AND $g^{(N)}$ EXPRESSIONS}

The following are the explicit expressions of $\theta^{(2)}, \theta^{(3)}$, and $\theta^{(4)}$ as functions of the click and coincidence probabilities at the detectors:

$$
\begin{aligned}
& \theta_{[i j]}^{(2)}=\frac{1-P_{\operatorname{click}[i]}-P_{\operatorname{click}[j]}+P_{\operatorname{click}^{\otimes 2}[i j]}}{\left(1-P_{\operatorname{click}[i]}\right)\left(1-P_{\text {click }[j]}\right)}, \\
& \theta_{[i j k]}^{(3)}=\frac{1-P_{\operatorname{click}[i]}-P_{\operatorname{click}[j]}-P_{\text {click }[k]}+P_{\operatorname{click}^{\otimes 2}[i j]}+P_{\text {click }^{\otimes 2}[i k]}+P_{\text {click }^{\otimes 2}[j k]}-P_{\operatorname{click}^{\otimes 3}[i j k]}}{\left(1-P_{\text {click }[i]}\right)\left(1-P_{\text {click }[j]}\right)\left(1-P_{\operatorname{click}[k]}\right)},
\end{aligned}
$$




$$
\begin{aligned}
\theta_{[i j k l]}^{(4)}= & \frac{1}{\left(1-P_{\operatorname{click}[i]}\right)\left(1-P_{\operatorname{click}[j]}\right)\left(1-P_{\operatorname{click}[k]}\right)\left(1-P_{\operatorname{click}[l]}\right)}\left(1-P_{\operatorname{click}[i]}-P_{\text {click }[j]}-P_{\operatorname{click}[k]}-P_{\operatorname{click}[l]}+\right. \\
& \cdots+P_{\operatorname{click}^{\otimes 2}[i j]}+P_{\operatorname{click}^{\otimes 2}[i k]}+P_{\operatorname{click}^{\otimes 2}[i l]}+P_{\operatorname{click}^{\otimes 2}[j k]}+P_{\operatorname{click}^{\otimes 2}[j l]}+P_{\operatorname{click}^{\otimes 2}[k l]}+ \\
& \left.\cdots-P_{\operatorname{click}^{\otimes 3}[i j k]}-P_{\operatorname{click}^{\otimes 3}[i j l]}-P_{\operatorname{click}^{\otimes 3}[i k l]}-P_{\operatorname{click}^{\otimes 3}[j k l]}+P_{\operatorname{click}^{\otimes 4}[i j k l]}\right),
\end{aligned}
$$

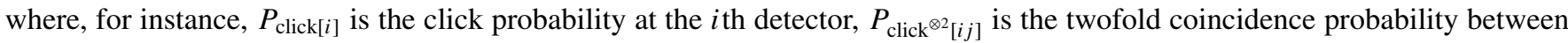
channels $i$ and $j$, and $P_{\text {click }^{83}[i j k]}\left(P_{\text {click }^{84}[i j k l]}\right)$ is the three- (four-)fold coincidence probability among channels $i, j, k(i, j, k, l)$. The latter probabilities are experimentally sampled from single channel detection $\left(N_{i}\right)$, the two- $\left(N_{i j}\right)$, three- $\left(N_{i j k}\right)$, and fourfold $\left(N_{i j k l}\right)$ coincidence rates, respectively, as $P_{\text {click }[i]}=N_{i} / N_{T R}, P_{\operatorname{click}^{\otimes 2}[i j]}=N_{i j} / N_{T R}, P_{\operatorname{click}^{\otimes 3}[i j k]}=N_{i j k} / N_{T R}, P_{\text {click }^{84}[i j k l]}=N_{i j k l} / N_{T R}$ and $N_{T R}$ is the rate of excitation events (the repetition rate of the excitation laser). Analogously, the $g$ functions are estimated as

$$
\begin{aligned}
& g_{[i j]}^{(2)}=\frac{P_{\operatorname{click}^{\otimes 2}[i j]}}{P_{\operatorname{click}[i]} P_{\operatorname{click}[j]}}, \\
& g_{[i j k]}^{(3)}=\frac{P_{\operatorname{click}^{\otimes 3}[i j k]}}{P_{\operatorname{click}[i]} P_{\operatorname{click}[j]} P_{\operatorname{click}[k]}}, \\
& g_{[i j k l]}^{(4)}=\frac{P_{\text {click }}^{\otimes 4}[i j k l]}{P_{\text {click }[i]} P_{\operatorname{click}[j]} P_{\operatorname{click}[k]} P_{\text {click }[l]}} .
\end{aligned}
$$

[1] G. Brida, M. Bondani, I. P. Degiovanni, M. Genovese, M. G. A. Paris, I. R. Berchera, and V. Schettini, Found. Phys. 41, 305 (2011).

[2] S. Ramelow, A. Mech, M. Giustina, S. Gröblacher, W. Wieczorek, J. Beyer, A. Lita, B. Calkins, T. Gerrits, S. W. Nam, A. Zeilinger, and R. Ursi, Opt. Express 21, 6707 (2013).

[3] S. Krapick, H. Herrmann, V. Quiring, B. Brecht, H. Suche, and C. Silberhorn, New J. Phys. 15, 033010 (2013).

[4] M. Förtsch, J. U. Fürst, C. Wittmann, D. Strekalov, A. Aiello, M. V. Chekhova, C. Silberhorn, G. Leuchs, and C. Marquardt, Nat. Commun. 4, 1818 (2013).

[5] N. Montaut, L. Sansoni, E. Meyer-Scott, R. Ricken, V. Quiring, H. Herrmann, and C. Silberhorn, Phys. Rev. Appl. 8, 024021 (2017).

[6] M. Oxborrow and A. G. Sinclair, Contemp. Phys. 46, 173 (2005).

[7] M. D. Eisaman, J. Fan, A. Migdall, and S. V. Polyakov, Rev. Sci. Instrum. 82, 071101 (2011).

[8] G. Brida, I. P. Degiovanni, M. Genovese, A. Migdall, F. Piacentini, S. V. Polyakov, and I. R. Berchera, Opt. Express 19, 1484 (2011).

[9] G. Brida, I. P. Degiovanni, M. Genovese, F. Piacentini, P. Traina, A. D. Frera, A. Tosi, A. B. Shehata, C. Scarcella, A. Gulinatti, M. Ghioni, S. V. Polyakov, A. Migdall, and A. Giudice, Appl. Phys. Lett. 101, 221112 (2012).

[10] A. J. Shields, Nat. Photonics 1, 215 (2007).

[11] M. Arita, F. Le Roux, M. J. Holmes, S. Kako, and Y. Arakawa, Nano Lett. 17, 2902 (2017).

[12] F. Diedrich and H. Walther, Phys. Rev. Lett. 58, 203 (1987).

[13] B. Lounis and W. E. Moerner, Nature (London) 407, 491 (2000).

[14] I. Aharonovich, D. Englund, and M. Toth, Nat. Photonics 10, 631 (2016).

[15] C. Kurtsiefer, S. Mayer, P. Zarda, and H. Weinfurter, Phys. Rev. Lett. 85, 290 (2000).

[16] A. Beveratos, R. Brouri, T. Gacoin, J.-P. Poizat, and P. Grangier, Phys. Rev. A 64, 061802 (2001).
[17] A. Beveratos, S. Kühn, R. Brouri, T. Gacoin, J.-P. Poizat, and P. Grangier, Eur. Phys. J. D 18, 191 (2002).

[18] D. G. Monticone, J. Forneris, M. Levi, A. Battiato, F. Picollo, P. Olivero, P. Traina, E. Moreva, E. Enrico, G. Brida, I. P. Degiovanni, M. Genovese, G. Amato, and L. Boarino, Int. J. Quantum Inf. 12, 1560011 (2014).

[19] D. G. Monticone, P. Traina, E. Moreva, J. Forneris, P. Olivero, I. P. Degiovanni, F. Taccetti, L. Giuntini, G. Brida, G. Amato, and M. Genovese, New J. Phys. 16, 053005 (2014).

[20] T. Schröder, S. L. Mouradian, J. Zheng, M. E. Trusheim, M. Walsh, E. H. Chen, L. Li, I. Bayn, and D. Englund, J. Opt. Soc. Am. B 33, B65 (2016).

[21] J. Forneris, P. Traina, D. G. Monticone, G. Amato, L. Boarino, G. Brida, I. P. Degiovanni, E. Enrico, E. Moreva, V. Grilj, N. Skukan, M. Jakšić, M. Genovese, and P. Olivero, Sci. Rep. 5, 15901 (2015).

[22] Single-Photon Generation and Detection Physics and Applications, edited by A. Migdall, S. V. Polyakov, J. Fan, and J. C. Bienfang (Academic, New York, 2013).

[23] M. Genovese, J. Opt. 18, 073002 (2016).

[24] I. I. Arkhipov, J. Peřina, O. Haderka, and V. Michálek, Opt. Express 24, 29496 (2016).

[25] C. J. Chunnilall, I. P. Degiovanni, S. Kück, I. Müller, and A. G. Sinclair, Opt. Eng. 53, 081910 (2014).

[26] P. Grangier, G. Roger, and A. Aspect, Europhys. Lett. 1, 173 (1986).

[27] R. Hanbury-Brown and R. Q. Twiss, Nature (London) 177, 27 (1956).

[28] E. A. Goldschmidt, F. Piacentini, I. R. Berchera, S. V. Polyakov, S. Peters, S. Kück, G. Brida, I. P. Degiovanni, A. Migdall, and M. Genovese, Phys. Rev. A 88, 013822 (2013).

[29] D. G. Monticone, K. Katamadze, P. Traina, E. Moreva, J. Forneris, I. Ruo-Berchera, P. Olivero, I. P. Degiovanni, G. Brida, and M. Genovese, Phys. Rev. Lett. 113, 143602 (2014). 
[30] A. Classen, F. Waldmann, S. Giebel, R. Schneider, D. Bhatti, T. Mehringer, and J. von Zanthier, Phys. Rev. Lett. 117, 253601 (2016).

[31] S. Oppel, T. Büttner, P. Kok, and J. von Zanthier, Phys. Rev. Lett. 109, 233603 (2012).

[32] H. Paul, P. Törmä, T. Kiss, and I. Jex, Phys. Rev. Lett. 76, 2464 (1996).

[33] L. A. Jiang, E. A. Dauler, and J. T. Chang, Phys. Rev. A 75, 062325 (2007).

[34] V. Schettini, S. V. Polyakov, I. P. Degiovanni, G. Brida, S. Castelletto, and A. L. Migdall, IEEE J. Sel. Top. Quantum Electron. 13, 978 (2007).

[35] A. Divochiy, F. Marsili, D. Bitauld, A. Gaggero, R. Leoni, F. Mattioli, A. Korneev, V. Seleznev, N. Kaurova, O. Minaeva, G. Gol'tsman, K. G. Lagoudakis, M. Benkhaoul, F. Lévy, and A. Fiore, Nat. Photonics 2, 302 (2008).

[36] E. A. Dauler, A. J. Kerman, B. S. Robinson, J. K. W. Yang, B. Voronov, G. Goltsman, S. A. Hamilton, and K. K. Berggren, J. Mod. Opt. 56, 364 (2009).

[37] D. Rosenberg, A. J. Kerman, R. J. Molnar, and E. A. Dauler, Opt. Express 21, 1440 (2013).

[38] K. Banaszek and I. A. Walmsley, Opt. Lett. 28, 52 (2003).

[39] M. J. Fitch, B. C. Jacobs, T. B. Pittman, and J. D. Franson, Phys. Rev. A 68, 043814 (2003).

[40] D. Achilles, C. Silberhorn, C. Śliwa, K. Banaszek, and I. A. Walmsley, Opt. Lett. 28, 2387 (2003).

[41] M. Avenhaus, K. Laiho, M. V. Chekhova, and C. Silberhorn, Phys. Rev. Lett. 104, 063602 (2010).
[42] M. J. Stevens, S. Glancy, S. W. Nam, and R. P. Mirin, Opt. Express 22, 3244 (2014).

[43] C. Thiel, T. Bastin, J. Martin, E. Solano, J. von Zanthier, and G. S. Agarwal, Phys. Rev. Lett. 99, 133603 (2007).

[44] O. A. Shcherbina, G. A. Shcherbina, M. Manceau, S. Vezzoli, L. Carbone, M. de Vittorio, A. Bramati, E. Giacobino, M. V. Chekhova, and G. Leuchs, Opt. Lett. 39, 1791 (2014).

[45] L. Lachman, L. Slodička, and R. Filip, Sci. Rep. 6, 19760 (2016).

[46] V. Acosta and P. Hemmer, MRS Bull. 38, 127 (2013).

[47] $g^{2}(0)$ below 0.5 is generally considered compatible with a single photon source, but we note that several different experimental situations may induce a $g^{2}(0)$ value far from 0 (as in our case), such as, e.g., a single emitter with some background noise, or few independent emitters with different emission probability, or an arbitrary number of correlated single emitters, etc.

[48] J. M. Binder, A. Stark, N. Tomek, J. Scheuer, F. Frank, K. D. Jahnke, C. Müller, S. Schmitt, M. H. Metsch, T. Unden, T. Gehring, A. Huck, U. L. Andersen, L. J. Rogers, and F. Jelezko, SoftwareX 6, 85 (2017), the software is available for download at https://github.com/Ulm-IQO/qudi.

[49] P. Obšil, L. Lachman, T. Pham, A. Lešundák, V. Hucl, M. Čížek, J. Hrabina, O. Číp, L. Slodička, and R. Filip, arXiv:1705.04472.

[50] L. Rondin, G. Dantelle, A. Slablab, F. Grosshans, F. Treussart, P. Bergonzo, S. Perruchas, T. Gacoin, M. Chaigneau, H.-C. Chang, V. Jacques, and J.-F. Roch, Phys. Rev. B 82, 115449 (2010).

[51] S. Pezzagna, B. Naydenov, F. Jelezko, J. Wrachtrup, and J. Meijer, New J. Phys. 12, 065017 (2010). 